\title{
A Specific Face Positioning Segmentation Research Based on Complex Background
}

\author{
Wang Jiang-Tao ${ }^{1+}$, Chen Duan-Sheng ${ }^{2}$ and Wang Jing ${ }^{2}$ \\ ${ }^{1}$ College of Engineering Technology, Yang-En University, Quanzhou 362014, China, \\ ${ }^{2}$ College of Computer Science and Technolgy, Huaqiao University, Xiamen 361021, China
}

\begin{abstract}
Facial gesture, light, expression, and other factors affect the formation of a complex background, under this wireless visual sensor network identification encountered unprecedented challenges. Traditional visual identification is required at node distribution is relatively complex environment, the uncontrollable factors transforming into a controllable, stable characteristic factors during the identification, thus leading to long recognition time, low efficiency. In this paper, the use of face recognition based on LBP wireless visual sensor network identity adaptive recognition method to get strong classifier cascade of underlying feature extraction, through the final Harr face cascade classifier human face testing, and the face region feature vector PCA dimension reduction, access to low-dimensional feature vector, the number of drop maintain, establish the simulation experiments, the feature matching facial feature library to complete the identification. Simulation results show that the proposed algorithm has high accuracy, effectively improve the recognition efficiency.
\end{abstract}

Keywords: wireless sensor, dimensionality reduction, feature matching, adaptive recognition.

\section{Introduction}

As wireless visual sensor network development and the application of information security people put forward higher requirements[1]. Licensing is one of the important means to ensure information security, it may be advantageous to protect users of the system have the corresponding authority[2, 3]. Identity adaptive knowledge is the key to the theoretical basis of authorization, recognition algorithm research topics have become the focus of scholars in the field, it has been more and more attention [4,5].

Identity recognition technology as a key development issues of network security, common identity recognition technology including face recognition, iris recognition and fingerprint recognition. Correlation algorithm achieved certain results. Among them, the literature[6] Proposed single feature identity recognition algorithm, based on a certain facial features to identify the identity of the algorithm has fast calculation speed, real-time advantage, but the affected light, rotation, etc., in a complex context, recognition rate will be reduced; literature[7] Proposed identity recognition algorithm combined features will not be fixed in accordance with the same characteristics as the principles together, and this algorithm is more efficient overall, but the information is too large, resulting in reduced real-time recognition; literature[8] SFM's proposed identity recognition algorithm, the first search match point, after SFM algorithm determined the three-dimensional coordinates of the feature points, using a multi-resolution image mosaic texture image forming algorithm, while the maps them to three-dimensional model, the algorithm increases the real sense, but a three-dimensional reconstruction of a large amount of computation, greatly affected the performance of its recognition.

Proposed LBP face discrimination in wireless visual sensor network identity characteristic adaptive algorithm to identify, by Harr face cascade classifier to detect human faces, human face region feature

\footnotetext{
+ Corresponding author. Tel: +17750783530

E-mail address: 14535325@qq.com
} 
vector PCA dimension reduction, for wireless local area face images, use LBP get facial feature information obtained facial feature information histogram statistics, get face LBP histogram, which will feature visual and wireless sensor networks signatures were human face match, to complete the identification of the identity. Experimental results demonstrate that the proposed algorithm has high recognition accuracy and efficiency.

\section{Visual Identity Adaptive Wireless Sensor Networks Recognition Principle}

Identity adaptive recognition through the inspection window in a customized location adjacent rectangular matrix of each pixel is obtained, at the same time seeking the difference, and then after the implementation of the recognition of the difference between the obtained sub-areas of the image.

In the identification process focused contain edge characteristics, central feature of the linear characteristic three samples, The total of 14 fundamental characteristic of the prototype.

If the detection window size, with quad rectangular description: $r=(x, y, w, h, \alpha)$, where in $(x, y)$ is used to describe the rectangular coordinates of the vertices; $(w, h)$ is used to describe the size of a rectangle; $\alpha$ is used to describe the rotation angle of the rectangle. the rectangle feature can be described as follows:

$$
\text { Feature }_{I}=\sum_{i \in I=\{1, \cdots, N\}} \omega_{i} \cdot \operatorname{recSum}\left(r_{i}\right)
$$

$\{1, \cdots, N\}, \omega_{i}$ continental is a graphical i weights, $\operatorname{recSum}\left(r_{i}\right)$ is all the pixels in the rectangle. Because the process of feature recognition calculation, the statistics need all the pixel values in a rectangle and makes training and testing efficiency is greatly reduced, therefore, we need to integrate special parameters to reduce property value calculating expenses, increase efficiency. Highlights include integral image angle is $0^{\circ}$ and $45^{\circ}$. For angle of $0^{\circ}$ is a characteristic square, integral image can be described:

$$
\operatorname{SAT}(x, y)=\sum_{x^{\prime} \leq x, y^{\prime} \leq y} I\left(x^{\prime}, y^{\prime}\right) \text { Feature }_{1}
$$

$I\left(x^{\prime}, y^{\prime}\right)$ is used to describe the pixels value of pointer $\left(x^{\prime}, y^{\prime}\right) . S A T(x, y)$ Expansion by formula (3) start, just press rows or columns to obtain a travers:

$$
\operatorname{SAT}_{1}(x, y)=\operatorname{SAT}(x, y-1)+\operatorname{SAT}(x-1, y)+I(x, y)-S A T(x-1, y-1)
$$

For matrix: $r=(x, y, w, h, 0)$, the pixels of the matrix and can be obtained target identification equation:

$$
\operatorname{recSum}(r)=\operatorname{SAT}(x-1, y-1)+\operatorname{SAT}(x+w-1, y+h-1)+I(x, y)-S A T(x-1, y+h-1)-S A T(x+w-1, y-1)
$$

The analysis equation, matrix $r$ 's $\operatorname{recSum}(r)$. Only time will be able to obtain integral image search, computational efficiency, while high recognition rate.

\section{Identity Recognition Algorithm LBP Face Recognition Adaptive Wireless Visual Sensor Networks}

\subsection{Face cascade classifier}

Harr Face cascade classifier using decision tree classifier manner in each period of the implementation of the search for the face, Increase the accuracy of inspection. In each period were performed using the discrete Ada boost algorithm sample training, a set of weak and strong classifiers were selected filter. Detailed exercise procedure is as follows:

- Consider the number of Sample image is N. $\left(x_{1}, y_{1}\right),\left(x_{2}, y_{2}\right), \cdots,\left(x_{n}, y_{n}\right)$, among them, $x_{i}$ used to describe the input sample image, $y_{i}=0$ is used to describe the classical mark, in case $y_{i}=0$, Representative of the detected non-face.in case $y_{i}=1$, The representative of the detected human face samples;

- Initialization processing by the following formula for weight: 


$$
\omega_{l, i}=\left\{\begin{array}{l}
\frac{1}{2 m}, y_{i}=0 \\
\frac{1}{2 l}, y_{i}=1
\end{array}\right.
$$

Where in, $m$ and $l$ were used to describe the number of the human face of the sample, $n=m+l$;

- By the following formula the weights are normalized:

$$
\omega_{t, i} \leftarrow \frac{\omega_{t, i}}{\sum_{j=1}^{n} \omega_{t, j}}
$$

Among them, $\omega_{t}$ is used to describe the statistical distribution;

- The final strong classifier can be described as:

$$
h(x)=\left\{\begin{array}{l}
1, \sum_{t=1}^{T} \alpha_{t} h_{t}(x) \geq \frac{1}{2} \sum_{t=1}^{T} \log \frac{1}{\beta_{t}} \\
0, \text { else }
\end{array}\right.
$$

Harr face cascade classifier strong classifier through the implementation of the sub-window to select image input face image after image fusion under the sub-window level in continued implementation of strong classifier selection, Finally, obtained with a high accuracy rate of face sub-window image.

\subsection{Face Image Processing PCA dimension reduction}

Harr face cascade respectively acquires face image face range message contains a plurality of Harr-like features, you want to increase the rear face alignment efficiency shall conclude the face range characteristic vectors,PCA dimension reduction governance,to obtain a low-dimensional feature vector and maintain lower number.And then the man's face in the field after the dimensionality reduction characteristic vector is normalized control, get the person's average face. PCA dimension reduction method of face range message is as follows:

- First, the face image of the sample by column expands into a one-dimensional vector, the calculation of their average image vector:

$$
m=\frac{1}{M} \sum_{i=1}^{M} x_{i}
$$

$x_{i}$ is used to describe a face samples Vector, $M$ is used to describe the total sample, $m$ is used to describe the average vector image, in the face image of the sample it is commonly referred to as the average face.

- Covariance matrix of training samples is calculated:

$$
C=\frac{1}{M} \sum_{i=1}^{M}\left(x_{i}-m\right)\left(x_{i}-m\right)^{T}
$$

For ease of calculation, the paper by the singular value decomposition theorems obtained eigenvalues, $\lambda_{i}(i=1,2, \cdots, n)$ used to be described, find the corresponding eigenvalues and orthogonal normalized eigenvectors.

- During the detection process, the input image can be projected to feature vector space face, there are:

$$
\alpha_{i}=\varphi_{i} \cdot\left(x_{i}-m\right)
$$

where, $\alpha_{i}$ is used to describe the projection coefficients corresponding to feature vectors, which can reflect the input image feature amount of the position. Then for any image, rectangular Feature reduce the dimension after dimension reduction feature can be described as: 


$$
\text { Pcafeatuer }=m+\sum_{j=1}^{k} \alpha_{j} \varphi_{j}
$$

\subsection{Algorithm implement}

LBP operator is a useful texture description operator, which uses the gray value associated with the size of the face image of each pixel with its neighbour points consisting of the binary-coded image of local texture space formed to express, not having a rotation denaturation and brightness invariant.

The name is a key point, then the size of the $3 \times 3$ window is called the gray this critical point is called the gray value of the eight-point threshold, and the associated implement contrast, if adjacent points than the center pixel value is small, the pixel position is 0 ; otherwise it is. The $3 \times 3$ binary field consisting of eight points depend on the specific rule changes for the line coding, you can get the LBP value point, this value reflects texture information in the region.

Paper completed PCA face detection and face region feature vector dimensionality reduction processing for wireless local area of the face image, which can operate LBP described as follows:

$$
\text { LbpFeature }\left(x_{c}, y_{c}\right)=\sum_{p=0}^{p-1} 2^{p} \cdot S\left(\text { PcaFeature }_{p}, \text { PcaFeature }_{c}\right)
$$

$\left(x_{c}, y_{c}\right)$ is used to describe the facial pixel image; PcaFeature ${ }_{c}$ is used to describe the dimensionality reduction Harr features; PcaFeature $_{p}$ used to describe the dimensionality reduction Harr feature of it's neighboring points; Dimensionality reduction Harr feature is used to describe the current pixel and its neighborhood point difference formula as follows:

$$
S(x)=\left\{\begin{array}{l}
1, \text { if } \quad x \geq 0 \\
0 . \text { else }
\end{array}\right.
$$

Face matching core idea is to be recognized by LBP facial features and all the characteristics of an average face library LBP features quite similar degree of variance, whether to identify suspicious face was left face characteristic library. Similarity variance by the following equation:

$$
d^{2}\left(L F_{c}, A L F_{n}\right)=\stackrel{b-1}{E}\left(L F_{c}(i), A L F_{n}(i)\right)
$$

$L F_{c}(i)$ is used to describe the face recognition LBP histogram bin characteristic features of the first block; $A L F_{n}$ and $A L F_{n}(i)$. The average face message and LBP histogram characteristics echoes; on behalf of the user to identify the person to be the face of LBP histogram bin. Similarity variance reduction, then the message with the face recognition of average face is more similar message echoes.

\section{Simulation Analysis}

In order to verify the proposed face recognition LBP wireless visual sensor network identity effectiveness of adaptive recognition algorithm needs to implement the relevant experimental resolution. Experiment traditional gait characteristics distinguish the characteristics of identity as a comparison algorithm simulation analysis.

\subsection{Database Settings}

Experimental data using UATV database, experiment database 300 contains images, depicted in Fig. 1 is part of a database that contains an image of the sample used in the experiment.

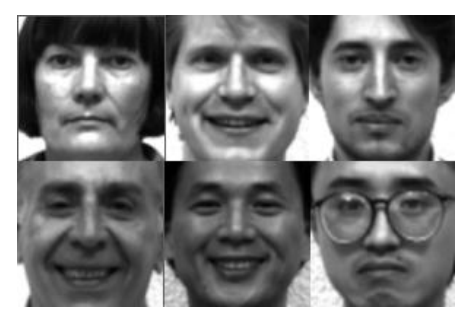

Fig. 1: Experimental sample image. 


\subsection{Algorithm recognition accuracy analysis}

From a total match score, the average recognition rate of two aspects of the implementation of this algorithm to identify test accuracy.Respectively, using this algorithm and the traditional algorithm for visual identity of wireless sensor networks in the implementation of adaptive identification, cumulative scores can be used to obtain matching Fig. 2 will be described.

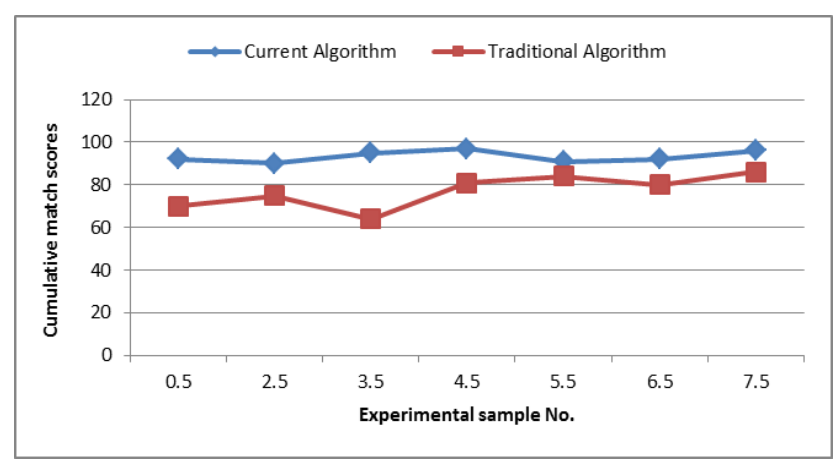

Fig. 2: Cumulative match scores compare two methods results.

Analysis Fig. 2 shows that the use of cumulative matching score algorithm was $93.1 \%$, the cumulative match scores traditional method is a $74.8 \%$ improvement compared to the traditional algorithm cumulative match scores improved $16.5 \%$. This is because it's article describes the algorithm effective facial features, improved identification correct classification rate to verify the accuracy of the algorithm.

To further verify the accuracy of the proposed method, the average recognition accuracy of the algorithm and the traditional algorithm were compared and analyzed, the results shown in Fig. 3.

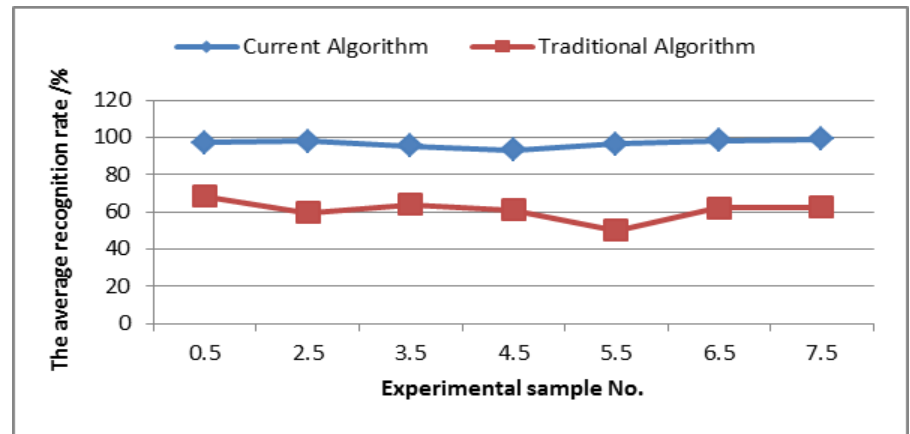

Fig. 3: Two ways the average recognition accuracy of the comparison result.

Analysis can be seen in Fig.3, the algorithm recognition accuracy rate of 94.3\%, traditional recognition algorithm $61.6 \%$ accuracy rate. Improved algorithm and traditional algorithm, using the average recognition accuracy of this algorithm a $31.3 \%$ increase compared to the traditional method, and the average recognition accuracy of the algorithm curve is more stable, indicating that the proposed method provides high recognition accuracy, but also has very high stability.

\subsection{Algorithm efficiency analysis}

On different types of child face image database, respectively algorithm and traditional identity recognition algorithms, efficiency of two algorithms were compared, the results shown in:

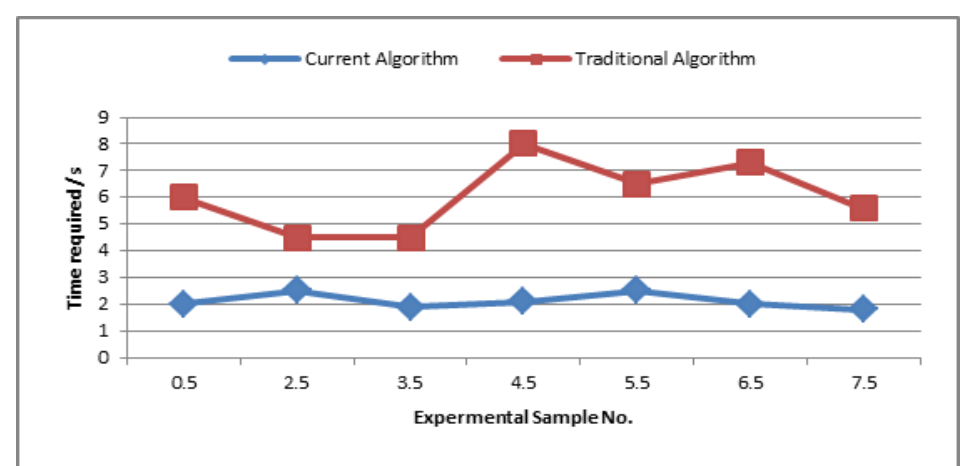

Fig. 4: Comparison of the results of the two algorithms efficiency. 
Analysis can be seen in Fig.4, for the same experimental sample, the algorithm time required for 2.4s, the time required for traditional methods of $6.3 \mathrm{~s}$. Using the time required for this algorithm compared to conventional algorithms to shorten the time required for the $3.9 \mathrm{~s}$, illustrated with traditional algorithms are compared, the higher the efficiency of the algorithm identification, is further evidence of the effectiveness of the algorithm.

\section{Conclusion}

LBP face recognition wireless visual sensor network identity adaptive recognition algorithm achieved strong association were cascaded devices, the use of high efficiency square integral image characteristics and the characteristics of the underlying method of extraction, after finally obtained Harr cascade classifier practice tests. Characteristics of the human face in the field of PCA dimension reduction control vector to obtain a low-dimensional features vector, and down to maintain the number of the person's average face. For wireless local area in the face image, given its operating LBP histogram statistics, get face LBP histogram, which feature wireless visual sensor networks and human facial feature database matching, complete identity of recognition. The simulation results show that:

- The cumulative improvement compared with the traditional algorithm matching algorithm scores improved by $16.5 \%$;

- The average recognition accuracy of this algorithm a $31.6 \%$ increase compared to the traditional method;

- The time required for the algorithm to shorten the time required for 4.1s compared to traditional methods.

\section{References}

[1] Liu Sha,Zhu Shuhua. Dynamic two-factor authentication scheme in a multi-server environment. Development and Research of Computers. 2015, 32 (4): 1119-1122.

[2] Zhu.P.Sh. Moving vehicle detection algorithm based on adaptive feature selection. Jishou University:Natural Science Edition. 2013, 34 (6): 47-52.

[3] Xu.B.K.Li.Chungui.Adaptive genetic algorithm mutation screening features face detection. Guang $x i$ University:Natural Science Edition. 2013, 24 (3): 73-77.

[4] Liu Zhonghua, Yao Nan,Liu Hong.Base on adaptive feature of human face recognition algorithm. Shanghai motor college. 2014, 4 (1): 224-228.

[5] Song Donghai,Chen Erhu. Edge-sensitive detection algorithm based on adaptive real palm Hog-LBP features. Computer and Digital Engineering. 2013, 41 (11): 1826-1828.

[6] Yuan Haiying,Hu Yu. Combined cycle smooth and adaptive features double threshold detection spectrum sensing algorithm. Computer Aided Design and Computer Graphics. 2013, 25 (4): 573-577.

[7] Wang Zhifeng,Mao. Qirong.Adaptive multi-fusion recognition algorithm based on grouping characteristics. Computer systems. 2014, 34 (3): 578-585.

[8] Zhang Fangqi,Hu Yong.Analysis and improvement user authentication scheme. Information Security and Communications Privacy. 2013, 24 (3): 73-77.

[9] Sun Pengbo,Zhang Wei,Wu Wangjun. CDMA2000 and other user identification method. Science Technology and Engineering. 2014, 14 (24): 80-83.

[10] Li Zhu, Hu Tao. Based on Adaptive LBP face recognition of identity verification. Computer Engineering and Science. 2014, 36 (11): 137-143. 